Inmigración y derecho al voto

Una investigación exploratoria en Santiago de Chile ${ }^{1}$

Borrador. Citar como:

Pérez, J.J.; Corbeaux, M.; and Doña-Reveco, C. 2021. "Inmigración y derecho al voto una investigación exploratoria en Santiago de Chile." SocArXiv. November 6. doi:10.31235/osf.io/h7abn.

José Joaquín Pérez

Sociólogo, Universidad Diego Portales, Chile.

Matías Corbeaux

Sociólogo, Universidad Diego Portales, Chile.

Cristián Doña Reveco (Autor correspondiente)

cdona@unomaha.com

Profesor Asociado de Sociología y Director, Office of Latino and Latin American Studies, University of Nebraska at Omaha. Investigador Asociado Instituto de Investigaciones Sociales, Universidad Diego Portales. Investigador Adjunto Centro de Estudios de Conflicto y Cohesión Social - COES - ANID/FONDAP/15130009, Chile.

\footnotetext{
${ }^{1}$ Esta investigación es parte del proyecto CONICYT+PAI/ Concurso Nacional Apoyo al Retorno de Investigadores/as desde el Extranjero. Convocatoria $2014+82140058$ proyecto: "Globalización Neoliberal, transformación social y migración internacional: Migrantes estadounidenses y españoles en Chile”. Fue financiada en parte gracias a un proyecto MiniCOES otorgado por el Centro de Estudios del Conflicto y la Cohesión Social, COES (CONICYT/FONDAP/15130009). Tiene como origen la tesis titulada "Participación política inmigrante en Chile: Condicionantes de la participación política activa. Una investigación exploratoria en la comuna de Santiago" (2017). Esta tesis fue realizada por los alumnos José Joaquín Pérez y Matías Corbeaux, bajo la tutela del Profesor Cristián Doña Reveco para optar al Título Profesional de Sociólogo de la Universidad Diego Portales.
} 


\title{
Inmigración y derecho al voto \\ Una investigación exploratoria en Santiago de Chile
}

\begin{abstract}
Resumen:
El objetivo principal de nuestra investigación es conocer las causas/factores que influyen en la intención de votar de los inmigrantes en la comuna de Santiago de Chile. Para lograr esto entrevistamos a veinte inmigrantes con derecho a voto en dos momentos; antes y después de las elecciones municipales de 2016. Logramos así comparar las respuestas teniendo las elecciones como eje del estudio. Realizamos un análisis de contenido de las respuestas para evaluar los discursos sobre la intención de votar. Podemos concluir que los factores que influyen en la decisión de votar - o no - por parte de los inmigrantes son variados. En particular mencionamos la falta de información sobre el derecho a votar en sí, la falta de interés de los candidatos en los votantes migrantes, y el no-reconocimiento de los migrantes como sujetos de derechos lo que resulta en un marcado desinterés en la política, de parte de los migrantes.
\end{abstract}

Palabras clave: Inmigración - Intención de Voto - Factores de influencia - Entrevistas - Santiago de Chile

Immigration and the Right to Vote

An Exploratory Study in Santiago, Chile

\begin{abstract}
:
The objective of our research is to analyze the causes and/or factors that influence the voting intentions of immigrants in the municipality of Santiago, Chile. To achieve this, we interviewed twenty immigrants who had the right to vote in two different periods. Before and after the October 2016 municipal elections. By doing this we were able to compare their answers having the elections as a pivotal point. We followed a content analysis of their answers to evaluate their discourses with regards to voting intentions. We conclude that the factors that influence immigrants' voting decisions are multiple. We center our conclusions in the following factors; lack of information migrants have on their electoral rights, lack of interest of candidates on migrants as a voting force, and lack of recognition from the State of migrants as legal subjects. These reasons produce high political indifference on immigrants.
\end{abstract}

Key words: Immigration - Voting intentions - Influencing factors - Interviews - Santiago, Chile 


\section{Introducción}

Uno de los principales temas de discusión pública en los últimos años en Chile ha sido el acceso que tienen los inmigrantes recientes a derechos sociales (Thayer et al, 2013; Stefoni, 2004). Sin cambiar la ley migratoria actual, vigente desde 1975, el Estado chileno ha ido poco a poco otorgando algunos derechos sociales a los nuevos inmigrantes. Específicamente, los migrantes tienen acceso a la atención de salud primaria y educación pública sin importar el estatus migratorio de la persona. Si bien el acceso a los derechos sociales este tema ha sido foco de importantes estudios, el análisis de este campo aún está incompleto (Agar, 2010; Cabieses, 2016; Stefoni et al, 2017; Riedemann y Stefoni, 2016; Correa et al, 2013). En particular, el estudio del acceso de los migrantes a los derechos políticos ha sido hasta ahora olvidado; pese a existir desde 1981 (Moreira, 2013; Doña Reveco y Sotomayor, 2017). En este trabajo presentamos algunos elementos exploratorios que permiten entender el acceso a la participación política de los inmigrantes en Chile, en particular en relación al derecho al voto.

La Constitución chilena, vigente con cambios desde 1981, establece en su artículo 14 que «Los extranjeros avecindados en Chile por más de cinco años, y que cumplan con los requisitos señalados en el inciso primero del artículo 13, podrán ejercer el derecho de sufragio en los casos y formas que determine la ley» (Chile, s.f.a). La ley que regula este derecho constitucional es la Ley 18.700 de 1988 promulgada aún en dictadura, específicamente en su artículo 60 (Chile, s.f.b). ${ }^{2}$ En el momento que se promulgó está ley residían en Chile menos de 100 mil personas nacidas en el exterior y representaban menos del $1 \%$ de la población total del país.

La migración hacia Chile comienza a crecer con el fin de la dictadura en 1991, principalmente por el retorno de exiliados. Sin embargo, es a partir de 1995 que se empieza a observar un flujo relevante de migrantes provenientes de países vecinos. Así, para el censo de 2002, los nacidos en el exterior casi doblaban a los del censo de 1992; llegando a casi 200 mil personas. El incremento de la migración desde ese momento ha superado todas las expectativas. De acuerdo al último censo válido, el de abril de 2017, se encontraban residiendo en Chile poco menos de 750 mil extranjeros. Esta cantidad equivale al 4,4\% de la población total del país; porcentaje que crece en más de tres puntos porcentuales desde 2002 (Doña y Levinson, 2012; Martínez Pizarro, 2005; DEM, 2015).

Por otra parte, la Ley $20.568,{ }^{3}$ de 2012 cambia las normas de participación electoral. Hasta antes de esta ley los mayores de 18 años tenían que ir personalmente a inscribirse en los registros electorales, para luego estar obligados a votar. Esta ley cambia esta situación, inscribiendo automáticamente a todos los mayores de 18 años, incluyendo a los nacidos en el exterior que llevan 5 o más años residiendo en el país. Así mismo consagra la voluntariedad del voto (Chile, s.f.c).

Hasta principios de 2016, el voto de los extranjeros no era un tema en la agenda pública asociada a la migración. Es a partir de marzo de ese año que el tema empieza a adquirir una cierta

\footnotetext{
${ }^{2}$ Articulo 60 Ley 18.700 «Son electores, para los efectos de esta ley, los ciudadanos con derecho a sufragio y extranjeros que figuren en los Padrones de Mesa y que tengan cumplidos dieciocho años de edad el día de la votación» (Chile, s.f.b).

${ }^{3}$ Ley 20.568: ley que regula la inscripción automática, modifica el Servicio Electoral y moderniza el sistema de votaciones. Comúnmente conocida como ley de "voto voluntario e inscripción automática." (Chile, s.f.c)
} 
presencia - limitada en todo caso en el contexto chileno - en la prensa nacional y en la opinión pública (Salazar, 2017a; Salazar, 2017b; El Mercurio, 2016; González, 2016). Las razones de la irrupción de este tema no están claras. Probablemente este asociado a las elecciones municipales que se iba a realizar en octubre de ese año, junto con la mayor visibilidad y concentración de los migrantes en ciertos municipios de la región Metropolitana - donde se ubica la capital de Chiley en unos pocos otros municipios del norte del país. Así mismo se da finalmente la consolidación de una serie de organizaciones de inmigrantes en un movimiento que se articula a partir de la necesidad que Chile tenga una nueva política migratoria basada en el acceso a los derechos de los migrantes. Esta relación entre acceso y derecho tiene consecuencias reales, tal como señalan Thayer et al., (2013), ya que al no reconocer a los migrantes como sujetos de derechos genera ciudadanías de primera y segunda categoría, donde los primeros (nativos) gozan de plena facultad de sus derechos sin mayores problemas relacionados al acceso, mientras que los segundos son considerados «subalternos» o culturalmente inferiores. Esto lleva a que se mantengan permanentemente es una situación de vulnerabilidad respecto de sus derechos sociales.

Así, en esta investigación pretendemos explorar los factores o causas que determinan la decisión de ejercer o no el derecho a voto de los inmigrantes en la comuna de Santiago. Para lograr esto realizamos y analizamos 40 entrevistas semiestructuradas con migrantes que ya tuvieran el derecho al voto y que residieran en la comuna de Santiago. Esta comuna la elegimos por ser la que tiene la mayor cantidad absoluta de migrantes en Chile, así como ser la comuna que tiene las comunidades migrantes más antiguas, y cuya elección de alcalde es una de las más políticamente relevantes a nivel nacional (Margarit y Bijit, 2014). Nuestros entrevistados los contactamos por el método bola de nieve y fueron entrevistados en dos procesos; antes de las elecciones municipales del 23 de octubre de 2016 y después de las elecciones municipales, teniendo como mediador este proceso electoral. Así, entrevistamos a las mismas personas dos veces, con un total de 20 entrevistas en la primera etapa y otras 20 en la segunda.

A continuación, presentamos los fundamentos teóricos más relevantes para nuestro estudio que tienen relación con la participación política de los migrantes y la relación entre inmigración y ciudadanía, en particular los estudios recientes sobre este tema en Chile. Seguidamente presentamos la metodología y muestra estudiada. En tercer lugar, presentamos cronológicamente el análisis de las entrevistas realizadas. Concluimos este artículo volviendo a los factores y condiciones que influyen en la participación política de los migrantes y su relación con la construcción de ciudadanías migrantes.

\section{Participación política y ciudadanía del inmigrante}

La participación política de los inmigrantes en el Estado moderno comenzó a través del ámbito laboral y la organización sindical a finales del siglo XIX. El derecho al voto, que se comienza a otorgar durante el siglo XX tiene como objetivo poder integrarlos e incluirlos a la comunidad de recepción (Calderón, 2006). Este proceso es considerado como una posibilidad de mejorar la democracia del país, ya que, al integrar a los inmigrantes a las decisiones de la sociedad, se les otorga un sentido de pertenencia al país que los acogió (Moreira, 2013). Sin embargo, son muy pocos los países que otorgan el voto irrestricto a sus inmigrantes en todos los niveles (municipal y nacional), por lo mismo, la escasa producción académica se concentra en Europa, y en algunos 
países latinoamericanos como Argentina y Uruguay (Pérez-Nievas et al., 2014; Birds et al., 2011; Moreira, 2013; Stuhldreher, 2012; Arrighi y Baübock, 2017)

En estos dos países, los estudios pretendían explicar la escasa participación de los extranjeros en las elecciones. En el caso de Argentina, Moreira (2013) concluyó que son pocos los votantes extranjeros que ejercen su derecho a la participación activa. Esto se da, por una parte, porque existe un procedimiento de registro que dificulta la participación. Igualmente, por otra parte, existe carencia de estrategias de información desde el Estado hacia los extranjeros sobre sus derechos y los procedimientos para ejercerlo. Estas conclusiones son similares a las del caso uruguayo. Según Stuhldreher (2012), existe poca participación electoral de los extranjeros con derecho a voto porque ninguna autoridad política ha promovido que los inmigrantes ejerzan su derecho a sufragio. También existen muchas trabas burocráticas para la inscripción del extranjero para votar, tales como el de tener una residencia legal o certificado de residencia. Al menos en este derecho, por tanto, los inmigrantes estarían alejados del ámbito de participación política, principalmente por la debilidad institucional y la poca información desde el Estado mismo. Así, estos estados han delegado la tarea de acoger al inmigrante a la ciudadanía misma, dejando al margen a las autoridades políticas (Thayer, 2016). Podemos hipotetizar, para el caso chileno, que ésta puede ser una de las causas importantes de la baja participación política de los inmigrantes.

Desde otra perspectiva, la inacción del Estado respecto del derecho al voto no sería casual. Si no más bien sería una acción intencionada, ya que «el voto es el instrumento de mayor poder para que las personas puedan incidir en las decisiones que afectan a toda la sociedad» (Aja y Moya, 2005, p. 66). Por lo que la preocupación de otorgar poder político a los inmigrantes no es solo el de entregarles responsabilidad civil, sino que es darles la posibilidad de poder decidir sobre los destinos de la nación, lo que para muchos significa, darle poder al otro en contra de los nacionales. El tema del acceso a derechos sociales de parte de los inmigrantes no es solo un tema de participación política, sino que es sobre todo un tema de políticas públicas que involucra también el acceso a salud y a la educación, entre otros. Así el acceso a los diferentes derechos, independiente de su índole, dependerá exclusivamente de qué tan informado está por una parte el inmigrante. Aún más importante es la información y disposición que tenga el encargado de poder generar el acceso al derecho, en el caso del derecho al voto, serían los encargados de conformar las mesas electorales.

La Constitución chilena, como planteamos anteriormente, equipara a los extranjeros con los ciudadanos en lo que respecta al voto. Los diferentes gobiernos desde 190, por su parte, les han ido otorgando otros derechos reservados para los ciudadanos. Sin embargo, el Estado chileno no reconoce a los extranjeros con derecho a voto como ciudadanos, lo que es una contradicción en sí. Por otra parte, la literatura académica sobre migración y ciudadanía coloca a la participación política - tanto a través del voto como otras formas - como una de las razones centrales por las cuales los migrantes pasan a ser ciudadanos (Bloemraad, 2006). Es así como en gran medida el caso chileno, y otros similares, da a los migrantes casi todos los derechos del ciudadano- excepto el ser elegido electoralmente y pertenecer a las fuerzas armadas — sin otorgarles el título ciudadano. Es más, en este sentido_-más allá que los ejerzan o no-los extranjeros residentes en Chile tienen, en el papel, más derechos ciudadanos que los chilenos que se encuentran encarcelados, por ejemplo. Con esto no argumentamos que efectivamente puedan ejercerlos, de hecho, como 
explicamos aquí hay barreras institucionales que impiden el acceso efectivo a este derecho. Es así como, antes de seguir con este análisis, es relevante discutir brevemente lo que entendemos por ciudadanía.

Como muchos de los conceptos de las ciencias sociales, el concepto de ciudadanía tiene numerosas definiciones y está en un continuo proceso de debate y construcción (Kivisto y Faist, 2007). La clásica definición de ciudadanía de Marshall (1950), nos permite entrar en la relación específica entre el migrante y el Estado-Nación que lo recibe, en este caso Chile. Así, Marshall propone que la ciudadanía está conformada por tres elementos:

a. Lo civil, que hace referencia a la libertad individual, la libertad de expresión, la libertad de pensamiento, la libertad a ejercer cualquier religión, la libertad a tener una propiedad privada y también tener libertad jurídica.

b. Lo político hace referencia entre otras cosas, al ejercer el derecho a sufragio para elegir a las autoridades políticas.

c. Lo social, que considera el bienestar económico y el derecho de poder relacionarse con otros, más allá de la esfera del trabajo.

Marshall, plantea, que el límite entre estos 3 elementos es difuso, todos tienen cierta relación y uno conlleva al otro. Esta definición pertenece a un período histórico específico, la post Segunda Guerra Mundial, y tiene relación con la expansión de derechos a las clases trabajadoras. Tiene como fondo denotar la inclusión de nuevos grupos nacionales en el contexto de la expansión del estado de bienestar de los países del norte de Europa. Se enfrenta muy rápidamente a la nueva era de las que convierte a esta parte de Europa de una zona de expulsión de migrantes a una zona de atracción de migrantes (Joppke, 2011). Es así como rápidamente la inclusión asociada a la idea marshaliana de ciudadanía se divide a una inclusión interior-los nacionales - y una exclusión exterior-los inmigrantes_como propone Brubacker (Joppke, 2011).

De acuerdo a lo que hemos planteado, entonces, los inmigrantes en Chile pasarían a ser inmediatamente ciudadanos. No por el título, sino por que caen inmediatamente en la inclusión interior al acceder a los derechos propuestos por Marshall. Sin embargo, en el caso chileno, legalmente los inmigrantes no son concebidos como ciudadanos, ya que al no tener la nacionalidad chilena no son nacionales. De esta manera, a pesar de tener la mayoría de los derechos y deberes de los chilenos, el no-reconocimiento trae consigo problemas de desigualdad social, económica, política y cultural. Estudiando específicamente el caso de la migración peruana, por ejemplo, Stefoni (2004) plantea que en la migración se reconocen ciudadanos de primera categoría y de segunda categoría. Los ciudadanos reconocidos como de primera categoría son aquellos que por el hecho de ser nacionales del Estado-Nación adquieren los derechos y deberes por naturaleza; es decir, son ciudadanos los chilenos nacidos en Chile o que mantengan alguna filiación con el país. Los ciudadanos de segunda categoría son aquellos que, si bien tienen todos los derechos de un ciudadano de primera categoría, no son nacidos en el país, o habiendo nacido en el exterior no tienen padre o madre chilenos. Resulta entonces paradójico que los inmigrantes, sin ser ciudadanos, puedan votar, ya que es una contradicción a los ideales de la democracia moderna y a los planteamientos de Marshall. 
Ciudadanía para el caso de los inmigrantes en Chile estaría, entonces, más cerca la noción de Pedroza, quien define este concepto como «una relación legal de individuos con un estado-nación específico, comúnmente determinada por requisitos que imprimen a esa relación una tinta de cultura, lenguaje y tradiciones legales y administrativas» (Pedroza, 2013, p. 3). Sin duda alguna, esta definición, teóricamente hablando, no excluye a los inmigrantes. En particular considerando que la gran mayoría de los inmigrantes recientes en Chile comparten con los nacionales el lenguaje, tradiciones legales e incluso ciertos aspectos culturales (Pellegrino, 2007).

La falta de una renovación en la política migratoria chilena, sin embargo, ha dejado «el proceso de incorporación a la suerte de las estrategias de los propios migrantes y a las respuestas que encuentra en la ciudadanía autóctona» (Thayer et al., 2013, p. 163). Existe por tanto una especie de abandono en los procesos de reconocimiento e integración en la comunidad de acogida, lo que produce que los nativos puedan actuar de forma hostil ante los procesos migratorios. De esta manera, Thayer y sus colegas (2013) mencionan que el reconocimiento normativo y social de los migrantes, es identificado como uno de los desafíos centrales de las democracias receptoras, de esta manera «una sociedad que se autodefine como democrática no puede sostener, a riesgo de caer en una contradicción en los términos, condiciones normativas y sociales que reproduzcan una "ciudadanía jerárquica", una "semi-ciudadanía"o un segmento de "ciudadanos de segunda categoría" en virtud de un acceso parcial a los derechos» (Thayer et al, 2013, p. 164).

En resumen, los extranjeros que tengan cinco años o más residiendo en Chile tienen automáticamente derecho a votar en cualquier elección. Considerando que la participación política por medio del voto ha sido estudiada - en países como Estados Unidos y Canadá entre otroscomo uno de los aspectos centrales para comprender la adquisición de ciudadanía, es interesante preguntarse ¿Cuáles son los factores que determinan la decisión de participar activamente en política de los inmigrantes en la comuna de Santiago de Chile? Estudiar exploratoriamente esta pregunta nos permite comprender situaciones de acceso a este derecho, relaciones entre el estado y los migrantes, características del proceso migratorio, entre otros temas.

\section{Métodos y muestra}

En esta investigación utilizamos una metodología cualitativa y exploratoria. Como señala Hernández Sampieri y sus colegas (2006), el enfoque cualitativo, tiene como propósito reconstruir la realidad tal y como la observan los actores sociales. De esta manera es un enfoque que se presenta como flexible y reflexivo a las exigencias del campo, flexible porque es capaz de adaptarse a las diferentes situaciones, tanto a las que el campo presenta como también a las que los actores sociales puedan presentar. Al mismo tiempo también es reflexivo, donde los investigadores no sólo son quienes ejecutan y analizan los datos, sino que son conscientes que forman parte de la investigación y de la producción de información (Mason, 2002). De esta manera, la construcción de conocimiento es situado y contextual, lo que permite la cooperación en la producción de conocimiento entre entrevistado y entrevistador (Mason, 2002). En esta investigación nos dimos cuenta de que, al entrevistar migrantes sobre el derecho al voto, podía darse el caso que les estuviéramos informando de ese derecho. Esto permitió que nos encontráramos frente a un experimento natural, que nos permitía evaluar en dos momentos la intención de votar y el acceso real al derecho al voto. 
Utilizamos la entrevista semi-estructurada, ya que en esta entrevista «es más probable que los sujetos entrevistados expresen sus puntos de vista en una situación de entrevista diseñada de manera relativamente abierta que en una entrevista estandarizada» (Flick, 2004, p. 89). Por otro lado, la investigación semi-estructurada «normalmente implica al entrevistador en un proceso tanto de construcción como de comprobación de modelos, tanto de construcción como verificación de teoría dentro de una misma sesión o de una serie de sesiones» (Wengraf, 2001, p. 4). Con este tipo de entrevista se puede seguir un cierto guion establecido por nosotros mismos. Así, no perdemos el foco de la investigación ni tampoco las consideraciones del marco teórico para poder guiar al entrevistado a ser más específico con sus respuestas. En nuestro caso, se busca saber precisamente cuáles factores condicionan que el sujeto participe en política o del por qué no participa en política. Utilizamos la técnica del análisis de contenido de la información, ya que esta nos permitía estudiar la naturaleza y estructuras de los discursos de los entrevistados (Flick, 2004; Wengraf, 2001).

Los criterios de selección de la muestra son bastantes claros, ya que están establecidos por la misma ley de votaciones citada anteriormente. De esta manera el criterio de selección se resume en que los inmigrantes deben: 1) tener 18 años de edad y 2) acreditar 5 años de residencia en el país. Considerando esto, todos los inmigrantes que no cumplan con estos dos aspectos quedaron fuera de la muestra ya que no tendrían derecho a participación activa en Chile. Esta investigación la realizamos el año 2016, ya que éste fue un año electoral, donde se realizaron elecciones para elegir alcaldes y miembros del consejo comunal en todas las regiones de Chile. Esto nos permitió evaluar el acceso al voto en dos momentos, uno antes de la elección y uno después de la elección. Las elecciones en sí se realizaron el 23 de octubre de 2016.

Para acceder a la muestra, utilizamos un mapeo de población inmigrante de la comuna de Santiago realizado por el departamento de estadísticas generales de la Ilustre Municipalidad de Santiago en el año 2013. En particular utilizamos mapas de densidad inmigrante para conocer las principales zonas de residencia dentro de la comuna. Este mapa. sin embargo, no discrimina entre aquellos que tienen y no tienen derecho al voto, por lo que aplicamos los criterios de selección de datos durante el trabajo de campo.

En general la migración reciente se ha concentrado en el sector poniente y norte de la comuna. En particular en los barrios Yungay y Balmaceda; ambos antiguos polos de comercio donde se encuentran la mayoría de los cites y conventillos que datan de la época colonial y que hoy son ocupados por inmigrantes (RIMISP, 2014). Debido a que los países de origen y características de los inmigrantes residentes en estos dos barrios son muy similares, elegimos el barrio Yungay por ser un barrio migrante consolidado con anterioridad a Balmaceda. Por otra parte, elegimos el barrio Bellas Artes ubicado al nororiente de la comuna para realizar una contraposición entre ambos. Así, mientras en el barrio Yungay se ven inmigrantes provenientes de países limítrofes y con menor calificación, en el barrio de Bellas Artes viven inmigrantes de alta calificación y con título profesional; muchas veces de países del Norte Global. A pesar de las distintas características socioeconómicas, prácticamente no existió diferencia sobre el poco interés en la política entre los inmigrantes del barrio Yungay y los del barrio Bellas Artes. 
Finalmente realizamos 20 entrevistas en total, 10 en cada barrio, principalmente en los lugares de trabajo de los migrantes. Las entrevistas ocurrieron en dos periodos de tiempo; primero en el período pre eleccionario de julio a mediados de octubre y luego post elecciones entre octubre y diciembre de 2016. Para el análisis final eliminamos una entrevista de la segunda etapa ya que no nos fue posible encontrar al entrevistado.

\section{Resultados}

Un primer resultado general que sobresale de las entrevistas es la relación contrapuesta entre motivo migratorio y participación electoral. Nuestros entrevistados argumentan que ellos estos vienen solo a trabajar, creen que su vida económica no va a cambiar con los derechos políticos. Durante las entrevistas nos pedían que esta no fuera muy larga, ya que ese era tiempo sin trabajar, tiempo sin ganar dinero. Para la mayoría de los entrevistados la política y los derechos políticos quedan en segundo plano. Esto no es necesariamente extraño. Hasta 2016 la migración hacia Chile era una migración principalmente laboral, formada por aquellos que había sido dejados de lado por el sistema económico de sus países. Así estos migrantes conectaban la falta de trabajo en el país de origen con políticas económicas llevadas a cabo por políticos definidos por ellos como corruptos. Esta falta de confianza hacia la política y los políticos se traspasa hacia el país receptor, separando el trabajo diario-para sobrevivencia y remesas - de la acción política. Como nos dijeron estos inmigrantes peruano y ecuatoriano:

Yo creo que los inmigrantes están más interesados en el trabajo que en la política, no les interesa informarse" (Inmigrante peruano, residente del barrio Yungay) ...la verdad tampoco me interesa la política ni tampoco los políticos (Inmigrante ecuatoriano, residente del barrio Yungay)

Más allá de este primer resultado, el tema principal que surge de las entrevistas es la falta de información en el acceso al derecho al voto. En las voces de los entrevistados es posible observar tres entradas específicas a este problema. Por una parte, el acceso es limitado por los mismos políticos que consideran poco relevantes a los migrantes en términos electorales. En segundo lugar, la falta de información desde candidatos y del Estado sobre este derecho y, en tercer lugar, la falta de políticas públicas y el no reconocimiento del migrante como sujeto de estas políticas. A continuación, presentamos los resultados por orden cronológico de las entrevistas, es decir primero las entrevistas del periodo pre eleccionario, seguidas de las entrevistas del período post elecciones.

\section{Primer periodo de entrevistas: julio a mediados de octubre de 2016}

Las entrevistas nos muestran que los inmigrantes tienden a no ejercer su derecho a voto por diferentes razones. Por un lado, de manera similar al caso uruguayo, los entrevistados perciben una poca preocupación de los políticos por incluir a los inmigrantes en sus discursos y en la elaboración de políticas públicas (Stuhldreher, 2012). Casi un tercio de nuestros entrevistados ${ }^{4}$ no les motiva ir a votar porque creen que su calidad de vida no va a cambiar si eligen a un político u

\footnotetext{
${ }^{4}$ No se debe asumir representatividad de estos resultados, son sólo una guía para describir las opiniones mencionadas en las entrevistas.
} 
a otro, creen que los discursos políticos y las propuestas de los candidatos no van dirigido hacia ellos. De hecho, una mayoría de los entrevistados no saben si los candidatos tienen propuestas para los inmigrantes. O los candidatos no han sabido transmitir a la comunidad migrante sus políticas sobre la inmigración o simplemente no están en sus planes generar este tipo de políticas públicas. Un argumento que proponen los entrevistados, ejemplificado en la cita siguiente, es que los políticos no los toman en cuenta ya que representan un porcentaje de voto muy bajo y no les serviría para ser electos.

¿Tú sabes si algún candidato de por acá tiene propuestas para los inmigrantes?

Mira, yo nunca he visto nada. Incluso nunca me llegó el tema del voto, porque yo creo que también si llegara por último alguien ha decirle a los inmigrantes a partir de ahora [...] Yo creo que justamente donde los extranjeros no saben que pueden votar, no es el público fuerte todavía. Yo creo que en cuatro años más, como está llegando mucha gente, va a ser como que va a haber alguien que va a tirar muchas cosas para los extranjeros, como está llegando mucho. (Inmigrante brasilero, residente del barrio Bellas Artes)

Un análisis de los votantes posibles disputa el argumento de la poca relevancia del voto migrante. Según la encuesta CASEN del año 2015, los inmigrantes en Chile equivalen al 2,4\% de la población. También se puede establecer, según datos del SERVEL, que en Chile existen 14.121.316 personas con derecho a voto, de estos, 238.716 son inmigrantes, es decir, los inmigrantes representan casi un 1,7\% de los electores. Si bien este número es poco relevante en una elección presidencial ${ }^{5}$, la concentración de los migrantes en comunas específicas lleva a pensar que a nivel comunal si pueden tener una influencia real. Esto se puede observar usando como ejemplo la votación del año 2012 en las cuatro comunas del gran Santiago que han recibido desde 1995 la mayor cantidad de inmigrantes. Es importante destacar que el stock de migrantes en el 2012 era significativamente inferior al stock en 2016 (DEM, 2015).

\section{Gráfico 1}

Votación elecciones comunales 2012 y voto inmigrante desconocido. Comunas seleccionadas

\footnotetext{
${ }^{5}$ Dicho esto, la elección presidencial más reñida de la historia reciente de Chile, la segunda vuelta entre Ricardo Lagos y Joaquín Lavín en el año 2000 se decidió por poco más de 200 mil votos.
} 


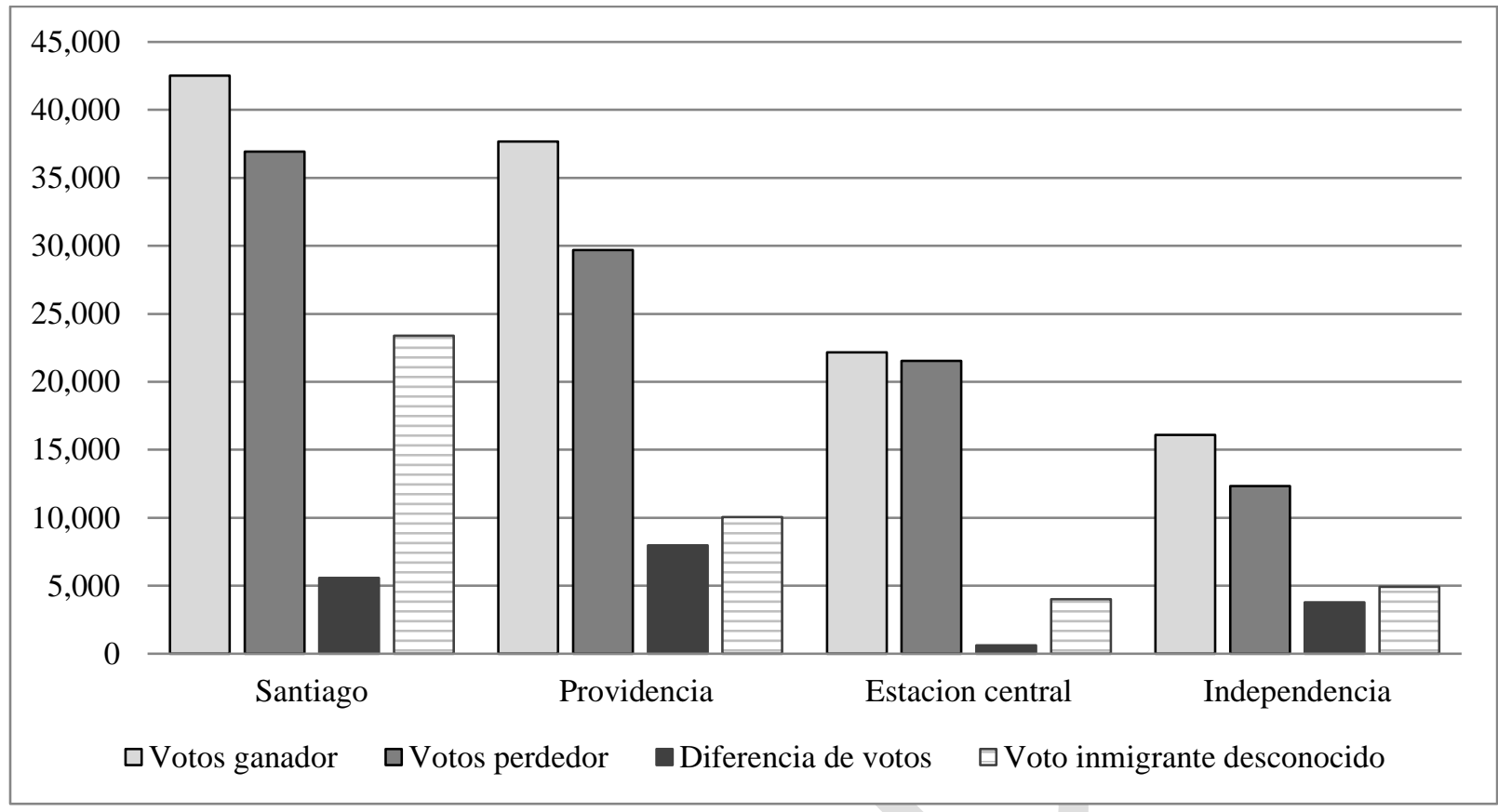

Fuente: Gráfico de elaboración propia, en base a información obtenida del SERVEL (https://www.servel.cl/)

En este gráfico podemos observar la cantidad de votos que recibió el candidato ganador, la cantidad de votos del candidato perdedor, la diferencia entre estos dos votos y la cantidad de inmigrantes que votan en cada comuna, voto hasta ahora desconocido. Como se observa la diferencia de votos entre ganador y perdedor es significativamente inferior a la cantidad de votos inmigrantes en cada comuna. Si bien no es posible sumar directamente los votos migrantes a uno u otro, podemos hipotetizar que la participación política de los migrantes puede incidir directamente en la elección de uno u otro candidato.

Por otra parte, y similar al caso argentino, una segunda fuente de exclusión es la falta de información relativa a la participación política por medio del voto (Moreira, 2013). La mayoría de los inmigrantes entrevistados no solo no conocían su derecho a votar; ningún candidato ni el Estado les habían informado de este derecho. Esta desinformación está ejemplificada en la siguiente cita:

\section{¿Usted ha votado alguna vez en Chile?}

No (...) Porque antes no podía. No sabía, si podía o no podía. Tú dices que sí, pero antes no. Tú dices que desde el 2010 pero yo no creo porque las últimas elecciones ¿fueron hace cuánto? (Inmigrante ruso, residente del barrio Bellas Artes)

La respuesta del inmigrante ruso demuestra una clara falta de información de los derechos que el Estado les otorga a los extranjeros. La situación es similar en la mayoría de los inmigrantes; quienes no creían que tenían el derecho a sufragio:

\section{¿Ha votado usted alguna vez en Chile? \\ No \\ ¿Por qué no le interesa o porque no sabía?}


Es que no sabía, con mis compañeros decíamos que no nos compete. (Inmigrante peruano, residente del barrio Yungay)

Como se ve anteriormente, en algunos casos llega a tal el desconocimiento de los derechos que tienen los inmigrantes, que entre ellos mismos se comunican la idea de que no pueden votar. No existe diferencia entre los barrios estudiados en la comuna de Santiago sobre la poca información de los derechos que tienen los extranjeros. Esta falta de reconocimiento del inmigrante como un sujeto con acceso a derechos provoca una discriminación o jerarquización de ciudadanías de primera categoría para los nacionales y de segunda categoría para los inmigrantes (Stefoni 2004).

Una tercera forma de exclusión la podemos observar utilizando el argumento marshaliano de ciudadanía y derechos presentado anteriormente. Si bien los inmigrantes cuentan con plenas facultades para acceder a los derechos de un ciudadano chileno, en la práctica, estos no son reconocidos como tal. Esto implica una contradicción entre la teoría de ciudadanía y la práctica del reconocimiento del estatus. Por otro parte, implica también una contradicción al cuerpo legal chileno. Como mencionamos anteriormente, si bien el Estado les otorga derechos ciudadanos no los reconoce como tales. Esto implica que en distintas oportunidades el acceso no sólo al derecho político de ejercer el voto, sino que a derechos de salud, educación y vivienda les sean negados, lo que produce discriminación y hostilidad constante hacia los inmigrantes. Esto lleva a un falso reconocimiento de parte del estado que reproduce condiciones de desigualdad entre los nacionales y los inmigrantes, dificultando su incorporación a Chile (Thayer, 2013).

Igualmente, esta contradicción entre la teoría y la práctica del ser ciudadano contribuye directamente a poner en discusión los reales fundamentos de la democracia. Como señalan Thayer y sus colegas (2013), el reconocer el sentido de la incorporación de los inmigrantes a la sociedad de acogida, puede ser visto como un aporte a aquellos que hace posible la democracia, por el contrario, su no reconocimiento, aporta a aquello que hace "imposible" un sistema democrático. En nuestra primera ronda de entrevistas observamos que once inmigrantes declaran no votar y uno no sabe. Los argumentos que sustentan el no votar están directamente relacionados al falso reconocimiento como ciudadanos activos de la sociedad civil particularmente al no incluirlos en sus programas políticos. En general, la mayoría de los entrevistados dicen que no saben de políticas públicas referidas a la inmigración por parte de los candidatos. En este sentido, el no incorporar a los migrantes a la discusión sobre programas locales, como educación, salud, vivienda, o más aún convivencia, limita directamente su participación política. Así se configura una omisión a la condición de ciudadanía y un problema profundo de reconocimiento de la población inmigrante, como un actor activo y potencial transformador de las elecciones a nivel municipal, como se demuestra en la siguiente cita:

¿Por casualidad tú sabes qué candidato tiene alguna propuesta que esté enfocada en extranjeros, o referidas a la inmigración?

No he escuchado a ninguno.

¿Por qué crees que no se da eso?

Yo creo que en el fondo no lo hacen porque en realidad no les interesa la problemática extranjera. (Inmigrante colombiano, residente del barrio Yungay) 
Teóricamente, en una democracia los políticos deberían tender a interesarse por todos los ciudadanos, ya sea por razones ideológicas o instrumentales para conseguir más votos. A este respecto, para los migrantes, los políticos no se preocupan por ellos profundizando el falso reconocimiento y la creación de "ciudadanías de segunda categoría". Nuestros entrevistados consideran que para lograr un auténtico reconocimiento (Thayer, 2016) es necesario que los políticos sepan que la categoría extranjero o inmigrante es una categoría relevante, como se demuestra en los siguientes ejemplos:

¿Cómo cree usted que tiene que ser un candidato para que atraiga el voto extranjero? Que apoye primeramente como migrantes, luego como ser humano. Que entienda que migrar no es un delito, es un derecho. Si es así tendrá nuestro apoyo. Nosotros vinimos a Chile porque está en vías de desarrollo y nosotros somos parte de la solución, no del problema. (Inmigrante dominicano, residente del barrio Yungay)

Porque yo pienso que cualquier candidato que esté interesado, y se enfocara en la problemática de los extranjeros, te aseguro que sale electo. Esto porque hay muchísimo extranjero. (Inmigrante Colombiana, residente barrio Bellas Artes)

Los inmigrantes sienten que en la práctica no tienen los mismos derechos que los chilenos. El responsable de otorgar este derecho es el Estado. Sin embargo, como observamos, no basta con crear el acceso en el papel, también es necesario dar a conocer, promocionar y facilitar el acceso al voto.

\section{Segundo periodo de entrevistas: Fines de octubre a noviembre de 2016}

La elección del domingo 23 de octubre, junto con el diseño metodológico, nos permitió contar con una especie de experimento natural. Así, asumimos que al haberle entregado nosotros la información a nuestros entrevistados que podían ejercer el voto, estos iban a efectivamente votar. El segundo proceso de entrevistas sirvió además para profundizar en los temas desarrollados en la primera entrevista. De los veinte entrevistados originales, sólo pudimos encontrar a diecinueve, por lo que presentamos este número de entrevistas.

Interesantemente, sólo una minoría ejerció su derecho a voto en las elecciones municipales de 2016. De los diecinueve entrevistados, catorce declararon no votar. De estos catorce, ocho corresponden al barrio Bellas Artes, mientras que seis al barrio Yungay. Respecto de las razones de no votar, la mitad mencionó motivos personales como trabajo, vida social y trámites; cerca de un tercio no voto por no conocer a los candidatos, mientras que dos declararon que los candidatos no valían la pena. De aquellos cinco que votaron, cuatro declararon que lo hicieron por cumplir con su deber cívico, mientras que el restante lo hizo por conveniencia. Pese a esta baja participación, una mayoría de los entrevistados (doce de los diecinueve) considera que existe un impacto del voto inmigrante en la configuración de políticas públicas. La pregunta sigue siendo por qué, pese a ser informados de sus derechos, y de considerar relevante su participación, los inmigrantes que votaron fueron sólo un cuarto del total de los entrevistados.

Como mencionamos anteriormente, la poca participación está asociada más ampliamente con la 
fuente de información al acceso al derecho al voto. Nuestros entrevistados argumentaron que sólo recibieron la información de parte nuestra, no de una institución o de un oficial del Estado de Chile. Los ejemplos siguientes hacen referencia directa a esa falta de información al momento de decidir votar:

No veo mucha televisión, pero nunca dijeron que ustedes pueden votar. Yo solamente he escuchado de ti. Si a mí me dicen diez veces que puedo votar, en realidad quizás vaya a votar, pero nunca lo escuche. (Inmigrante ruso residente del barrio Bellas Artes)

Yo creo que porque no sabían que podían votar. Yo por ejemplo supe por ustedes, y lo otro es que mucha gente puede decir como que no es mi problema, que no es mi país, pero eso no es mi ideología. Yo creo que hay mucha gente que piensa así. (Inmigrante peruano residente del barrio Yungay)

La gran mayoría de los inmigrantes que no votaron, al no sentirse incluidos, tienen la idea de que falta inclusión:

¿Por qué cree que los extranjeros no fueron a votar?

Por falta de información igual que yo. No tanto de interés porque tal vez falta inclusión. (Inmigrante ecuatoriano residente en Yungay)

La falta de información provoca falta de interés. Por otro lado, los políticos tratan al inmigrante como un chileno cualquiera, no consideran otros factores que pueden afectar la calidad de vida de los extranjeros. Los inmigrantes tienen una realidad distinta, tal como afirman los entrevistados:

¿Cree, por ejemplo, que mientras más voto extranjero haya, mejor calidad de vida pueden ustedes tener?

No creo. Las leyes pueden en parte, por ejemplo, a visas de trabajo, contrato de trabajo. Pero en la calidad de vida, los arreglos de casas y ese tipo de cosas. Como hay distintas necesidades, arrendar una pieza y los precios no conviene. (Inmigrante peruana, residente barrio Yungay)

En esta segunda ronda de entrevistas, los inmigrantes sostienen además que ellos no son parte del discurso de los políticos. Esta falta de reconocimiento se da por la incapacidad del Estado de proponer políticas de inclusión. Para éste, no es su tarea insertar al inmigrante en la sociedad. Como sostiene Thayer y sus colegas «el proceso de incorporación [es dejado] a la suerte de las estrategias de los propios migrantes y a las respuestas que encuentra en la ciudadanía autóctona» (Thayer et al., 2013p. 163). Esta idea se complementa con lo que dijeron nuestros entrevistados:

¿Por qué cree que los extranjeros no votan?

Yo creo que porque los políticos no se dan a conocer, no dan propuestas como para que uno salga y voten por ellos. No están ni ahí con los extranjeros yo creo. (Inmigrante colombiana residente del barrio Bellas Artes) 
Esta entrevistada, reafirma la idea de que son las autoridades locales y sobre todo las conexiones que realizan los inmigrantes con sus vecinos, el vehículo para lograr la integración a la sociedad. Esta idea es compartida en ambos barrios estudiados.

\section{¿Por qué cree usted que los extranjeros no fueron a votar?}

Porque como son migrantes tienen muchos problemas personales como para entender otra realidad en la que tienen que participar. (Inmigrante peruana residente del barrio Yungay)

En otras palabras, el Estado no ha asumido el problema migrante en Chile, no se lo ha apropiado de los problemas personales de los mismos inmigrantes. El potencial votante migrante, considera que tiene otras necesidades que los chilenos. No tiene sentido, que los políticos no tomen en cuenta a los extranjeros, como sujetos con problemas distintos. Las temáticas recogidas en la segunda tanda reafirman la falta de acceso al voto debido a la falta de información y a la no incorporación del migrante.

\section{Conclusiones}

Nuestro objetivo en este artículo ha sido presentar las posibles causas y/o factores que influyen en la decisión de votar de los inmigrantes en la comuna de Santiago. A partir de una investigación cualitativa y exploratoria hemos podido dar cuenta de tres causas por las cuales los inmigrantes no acceden al derecho al voto en Chile.

En primer lugar, no existe información oportuna desde el Estado sobre el derecho a voto de los inmigrantes. Por esto, los inmigrantes simplemente no conocen su derecho a la participación activa en política (derecho a voto). La poca información respecto a sus derechos produce que los inmigrantes se sientan desplazados de la categoría de sujetos de derecho, lo que termina finalmente con la decisión de no ejercer su derecho a sufragio, para evitar problemas en los locales de votación ante una negación de acceso al derecho por parte de los chilenos.

En segundo lugar, y por lo menos hasta las elecciones comunales de 2016, no existía intención política de incluirlos en programas de candidaturas, como tampoco en políticas públicas. Con respecto a esto es posible esbozar principalmente dos razones. Por un lado, la clase política no ve en la comunidad inmigrante un potencial nicho electoral. Los datos que presentamos contradicen esta afirmación. La comunidad inmigrante es un núcleo importante de voto en el Chile actual, principalmente para las elecciones municipales, pudiendo eventualmente influir en el resultado de una elección. Por otra parte, la no inclusión de la comunidad inmigrante en políticas públicas que realmente satisfagan sus problemáticas o en programas de candidaturas, se debe principalmente a que los políticos asumen a los inmigrantes como nativos; en este caso como chilenos. Así, el Estado chileno buscándole solución a la problemática migrantes desde una perspectiva hegemónica, sin considerar los conflictos cotidianos de la comunidad involucrada. Esto generalmente resulta en malas soluciones o incluso en acentúan los conflictos entre nacionales e inmigrantes (Antileo, 2013; Walsh, 2005). 
En tercer lugar, existe una clara contradicción en la definición de ciudadano bajo los parámetros del Estado moderno y el otorgamiento de derechos políticos a los inmigrantes en Chile. En este caso no se toma en cuenta al inmigrante para elegir a representantes públicos realmente, no hay interés en que estos hagan valer sus derechos, por esta razón, los extranjeros no son informados sobre su derecho a voto. No tiene utilidad alguna, otorgar derechos y no informar que se tienen, en la práctica es como si no lo tuvieran. Como se vio en la descripción de los datos, la mayoría de los inmigrantes no sabían que tenían el derecho a sufragio, claramente el que no tengan información y que ninguna autoridad haya informado a los inmigrantes sobre sus derechos, les provoca que exista poca motivación, porque se sienten marginados de un sistema político que no les informa oportunamente sus derechos.

Es por esta razón que Stefoni (2004) señala que los inmigrantes son reconocidos como ciudadanos de segunda categoría; a pesar de contar con todos los derechos de un ciudadano de primera categoría, en la práctica no es así. Los ciudadanos chilenos son continuamente informados sobre cuáles son sus derechos, en el caso del derecho a voto, el llamado político es para que el chileno vote, pero no existe un llamado al inmigrante para que vote. La información es desigual, y esto se traduce en un acceso desigual al derecho.

Respecto a los aspectos barriales de la investigación pudimos establecer que en líneas generales no existen grandes diferencias entre el barrio Yungay y el barrio Bellas Artes si de participación política se trata. Los datos obtenidos y su posterior análisis señalan que las causa o factores de influencia en la decisión de votar son los mismo es ambos barrios, es decir, poca información sobre el derecho a voto, baja inclusión en política públicas y programas de candidaturas, así como también la inexistencia del reconocimiento de sujetos de derechos o ciudadanos de primera categoría. La principal diferencia que encontramos está asociada a los motivos por los cuales decidirían votar. Mientras en el barrio Yungay la principal motivación para votar está asociada a conseguir mejoras económicas tanto para el barrio como personales, en el barrio Bellas Artes, sus residentes declaran votar por un deber cívico, aunque eventualmente no ejerzan su derecho a voto. Esto puede deberse al tipo de migración y a las características mismas de los barrios. Mientras que el barrio Yungay se configura como un sector de inmigración principalmente Latinoamericana y relacionada con trabajos de baja calificación, el barrio Bellas Artes se alza como uno de los sectores de la comuna de Santiago que ha vivido un fuerte proceso de gentrificación, donde los residentes inmigrantes, son principalmente del norte global.

Esta investigación nos permite pensar en un caso particular en el acceso a derechos, en particular el derecho al voto. Este es el caso del otorgamiento del derecho al voto sin ciudadanía, contrario a la mayoría de los países. Además, en el contexto de la inexistencia de una ley y de una política migratoria. De manera muy exploratoria resaltamos la necesidad que el Estado informe a todos los residentes de sus derechos de manera activa. Así mismo, este estudio nos lleva a pensar en nuevas formas de ciudadanías migrantes, en este caso una «ciudadanía marshaliana» sin nacionalidad. Esta idea nos permite ahondar en los procesos de inclusión de los migrantes en las sociedades receptoras, aportando a la creciente literatura sobre este tema (Joppke, 2011). Recordamos, sin embargo, el carácter exploratorio de esta investigación. Si bien hemos podido reconocer los factores que influyen en la decisión de los inmigrantes en ejercer su derecho a voto, es necesario 
complejizar aún más la investigación para así tener una mayor profundidad y aplicación teórica y práctica de sus resultados.

\section{Bibliografía}

Agar, L. (2010). Migraciones Salud y Globalización: Entrelazando Miradas. Santiago: Editorial Biplano, OIM/OPS/MINSAL.

Aja, E. y Moya, D. (2009). El Derecho de Sufragio de los Extranjeros Residentes. En: E. Aja, J. Eliseo, J. Arango y J. Oliver (Eds.). La inmigración en la encrucijada. Anuario de la inmigración en España 2007 (pp. 64-81). Barcelona: Bellaterra.

Antileo, E. (2013). Políticas indígenas, multiculturalismo y el enfoque estatal indígena urbano. Revista de Historia Social y de las Mentalidades, 17(1), 133-159.

Arrighi, J.T. y Bauböck, R. (2017). A multilevel puzzle: Migrants' voting rights in national and local elections. European Journal of Political Research, 56: 619-639. doi:10.1111/14756765.12176.

Birds, K., Saalfeld, T. y Wüst, A. (2011). The Political Representation of Immigrants and Minorities. Voters, parties and parliaments in liberal democracies. New York: Routledge.

Bloemraad, I. (2006). Becoming a Citizen: Incorporating Immigrants and Refugees in the United States and Canada. Berkeley: Univ. of California Press

Cabieses, B. (2016). Research on migration and health in Chile: pushing forward. Revista Médica de Chile, 144(8), 1093-1094.

Calderón, L. (2006). El estudio de la dimensión política dentro del proceso migratorio. Sociológica (México), 21(60), 43-74.

Chile (s.f.a). Constitución Política de la República de Chile. Biblioteca del Congreso Nacional de Chile. Recuperado de http://bcn.cl/1uva9

Chile (s.f.b). Ley Núm. 18.700 Ley Orgánica Constitucional sobre Votaciones Populares y Escrutinios. Biblioteca del Congreso Nacional de Chile. Recuperado de http://bcn.cl/1v16w

Chile (s.f.c). Ley Núm. 20.568 Regula la Inscripción Automática, Modifica el Servicio Electoral y Moderniza el Sistema de Votaciones. Biblioteca del Congreso Nacional de Chile. Recuperado de http://bcn.cl/1uxix

Correa, V., Bertolotto, I. y Musset, A. (2013). Geografías de la espera: migrar, habitar y trabajar en la ciudad de Santiago, Chile. 1990-2012. Santiago: Uqbar

DEM (Departamento de Extranjería y Migración, Chile) (2015). Migración en Chile, 2005-2014. Santiago: DEM. Recuperado de http://www.extranjeria.gob.cl/media/2016/02/AnuarioEstad\%C3\%ADstico-Nacional-Migraci\%C3\%B3n-en-Chile-2005-2014.pdf

Doña-Reveco, C. y Levinson, A. (2012). Chile: A Growing Destination Country in Search of a Coherent Approach to Migration. Migration Information Source. Recuperado de https://www.migrationpolicy.org/article/chile-growing-destination-country-searchcoherent-approach-migration

Doña-Reveco, C., y Sotomayor, P. (2017). ¿Por qué votan los inmigrantes? Participación electoral e integración entre migrantes en Chile. Blog Observatorio de Desigualdades, Universidad Diego Portales. Recuperado de http://observatoriodesigualdades.icso.cl/2017/12/por-quevotan-los-inmigrantes-participacion-electoral-e-integracion-entre-migrantes-en-chile/ 
El Mercurio (2016, junio 5). El voto de inmigrantes puede influir en el resultado electoral de 11 comunas. Recuperado http://www.economiaynegocios.cl/noticias/noticias.asp?id=259127

Flick, U. (2009). Introducción a la investigación cualitativa. Madrid: Ediciones Morata.

González, T. (2016, junio 13). El decisivo rol de los inmigrantes en las elecciones municipales. Diario U Chile Recuperado de https://radio.uchile.cl/2016/06/13/el-decisivo-rol-de-losinmigrantes-en-las-elecciones-municipales/

Joppke, C. (2011) Citizenship and Immigration. Cambridge: Polity

Kivisto, P. y Faist, T. (2007). Citizenship: Discourse, Theory, and Transnational Prospects. Malden, MA: Blackwell Pub.

Marshall, T.H. (1950). Citizenship and Social Class. Cambridge: Cambridge University Press.

Martínez Pizarro, J. (2005). Magnitud y dinámica de la inmigración en Chile, según el censo de 2002. Papeles de Población, 11(44), 109-147.

Mason, J. (2002). Qualitative researching. London: Sage Publications.

Moreira, C. (2013). Sufragio Transnacional: La experiencia del voto de los extranjeros en Argentina. Revista Encrucijada Americana, 5(2), 67-86.

Pedroza, L. (2013). Extensiones del derecho de voto a inmigrantes en Latinoamérica: ¿contribuciones a una ciudadanía política igualitaria? Una agenda de investigación. desiguALdades.net Working Paper Series 57, Berlin. Recuperado de https://refubium.fuberlin.de/handle/fub188/20118

Pellergino, A. (2007). Immigration et émigration en Amérique du Sud. Revue Hommes et migrations, 1270, 102-113.

Riedemann, A. y Stefoni, C. (2016). Sobre el racismo, su negación, y las consecuencias para una educación anti-racista en la enseñanza secundaria chilena. Polis, 42. Recuperado de http://journals.openedition.org/polis/11327

RIMISP (2014). Estudio y Diagnóstico del colectivo de migrantes residentes en la comuna de Santiago. Observatorio Santiago. Recuperado de http://www.observatoriosantiago.cl/wpcontent/uploads/2014/09/OP-ESTUDIO-DIAGNOSTICO-COLECTIVOMIGRANTES.pdf

Salazar, C. (2017a, marzo 29). Radiografía sobre participación política de los migrantes revela gran desinformación cívica. La Nación Recuperado de http://lanacion.cl/2017/03/29/radiografia-sobre-participacion-politica-de-los-migrantesrevela-gran-desinformacion-civica/

Salazar, C. (2017b, mayo 3). Cristián Doña, Sociólogo: El aporte del voto migrante podría ser decisivo en estas presidenciales. La Nación Recuperado de http://lanacion.cl/2017/05/03/cristian-dona-sociologo-el-aporte-del-voto-migrante-podriaser-decisivo-en-estas-presidenciales/

Hernández Sampieri, R., Fernández Collado, C., y Baptista Lucio, P. (2006). Metodología de la Investigación. México: McGraw-Hill Interamericana.

Margarit, D. y Bijit Abde, K. (2014). Barrio y población inmigrantes: El caso de la comuna de Santiago. Revista INVI, 29(81), 19-77.

Stefoni, C. (2004). Inmigración y Ciudadanía: La formación de comunidades peruanas en Santiago y la emergencia de nuevos ciudadanos. Política, 43, 319-336. 
Stefoni, C., Leiva, S. y Bonhomme, M. (2017). Migración internacional y precariedad laboral. El caso de la industria de la construcción en Chile. REMHU - Revista Interdisciplinar da Mobilidade Humana, 25(49), 95-112.

Stuhldreher, A. (2012). El sufragio transnacional: una aproximación al caso de Uruguay. Estudios Internacionales, 44(172), 7-28. doi:10.5354/0719-3769.2013.23575

Taylor, S. y Bogdan, R. (1996). Introducción a los métodos cualitativos de investigación. Barcelona: Paidos.

Thayer, E. (2016). Territorio, Democracia en crisis y Migración Transnacional. El estado chileno frente a la nueva pluralidad social. En: M. Guizardi (Ed.) Las Fronteras del Transnacionalismo (pp. 37-62). Santiago de Chile: Ocho Libros.

Thayer, E. (2013). Expectativas de reconocimiento y estrategias de incorporación: la construcción de trayectorias degradadas en migrantes latinoamericanos residentes en la Región Metropolitana de Santiago. Polis, 12(35), 259-285.

Thayer, E., Córdova, M.G. y Ávalos, b. (2013) Los límites del reconocimiento: migrantes latinoamericanos en la Región Metropolitana de Santiago de Chile. Perfiles Latinoamericanos 21(42.), 163-191.

Pérez-Nievas, S., Vintila, C., Morales, L., y Paradés, M. (2014). La representación política de los inmigrantes en elecciones municipales. Madrid: Centro de Investigaciones Sociológicas.

Walsh, C. (2005). Interculturalidad, conocimientos y decolonialidad. Signo y Pensamiento, 24, 3950.

Wengraf, T. (2001). Qualitative Research Interviewing. Biographic, Narrative and Semistructured Methods. London: Sage. 